\title{
Scanning Tunneling Microscopy and Spectroscopy of Wet-Chemically Prepared Chlorinated Si(111) Surfaces
}

\section{Supporting Information}

\author{
Peigen Cao, Hongbin $\mathrm{Yu}^{1}$, James R Heath ${ }^{*}$ \\ Division of Chemistry and Chemical Engineering, Noyes Laboratory, 127-72 \\ Kavli Nanoscience Institute \\ California Institute of Technology \\ Pasadena, California 91125 \\ ${ }^{1}$ Current Address: $\quad$ Department of Electrical Engineering \\ ERC 159, Mail Code 5706 \\ Arizona State University \\ Tempe, AZ 85287-5706 \\ * Corresponding author: \\ heath@caltech.edu
}




\section{Supporting Information}

Etch-pit and edge-atom coverage calculation steps: (An approximate method)

1. Average and background subtraction;

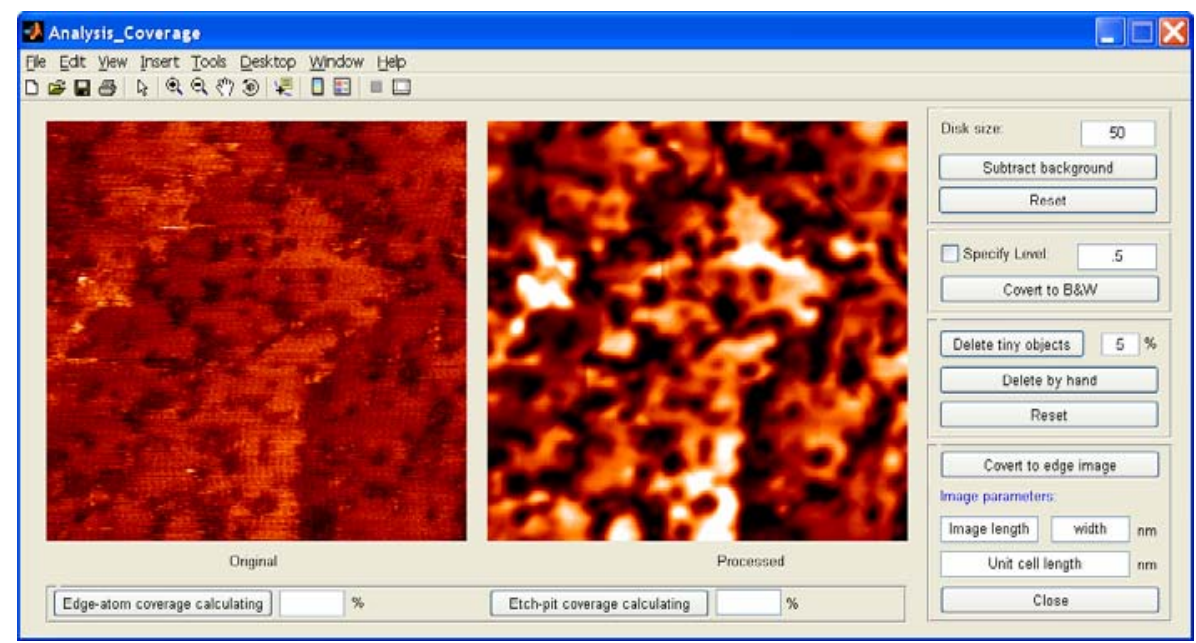

Note: different average level would lead to an error in the final result.

2. Converting to $\mathrm{B} \& \mathrm{~W}$ images;

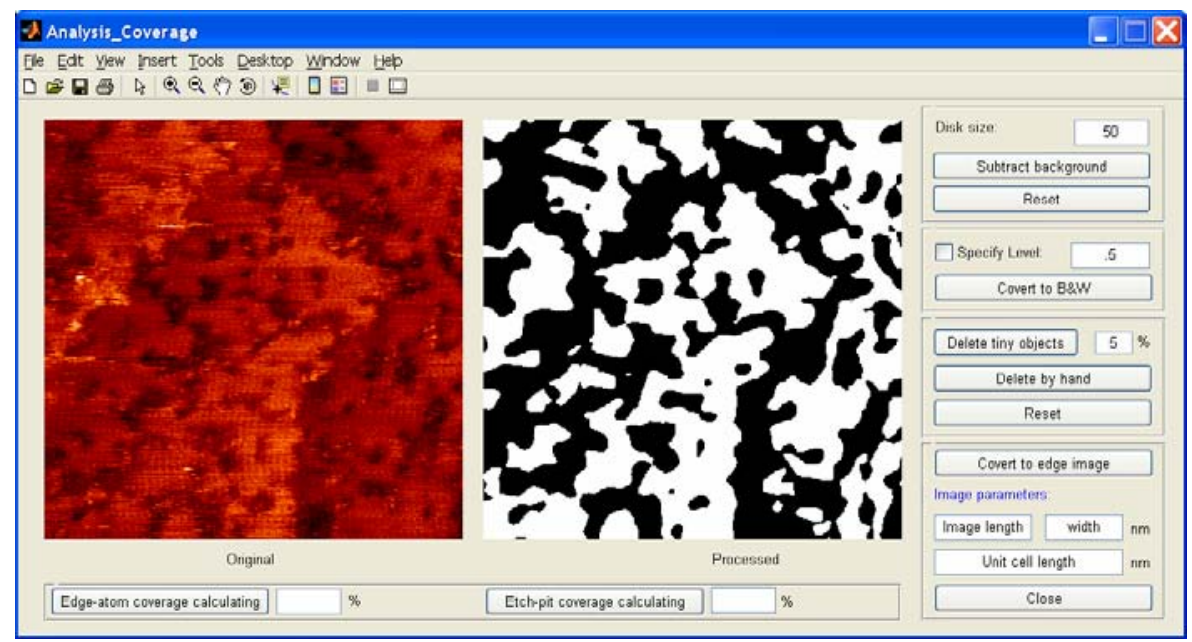

3. Converting to edge images; 


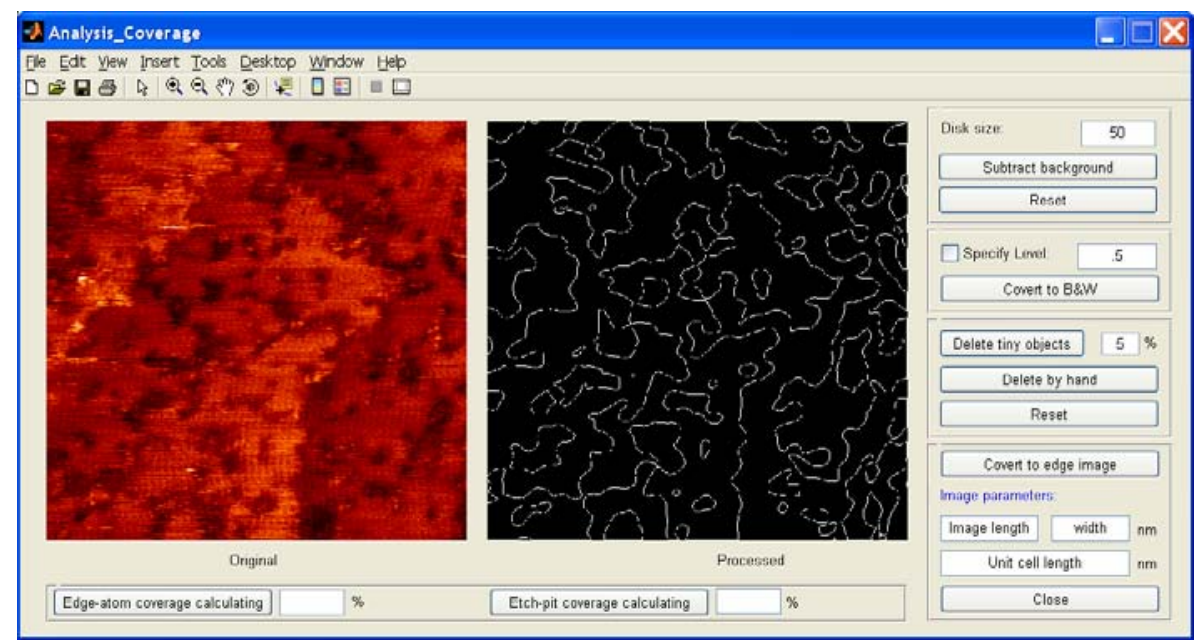

4. Calculating the results;

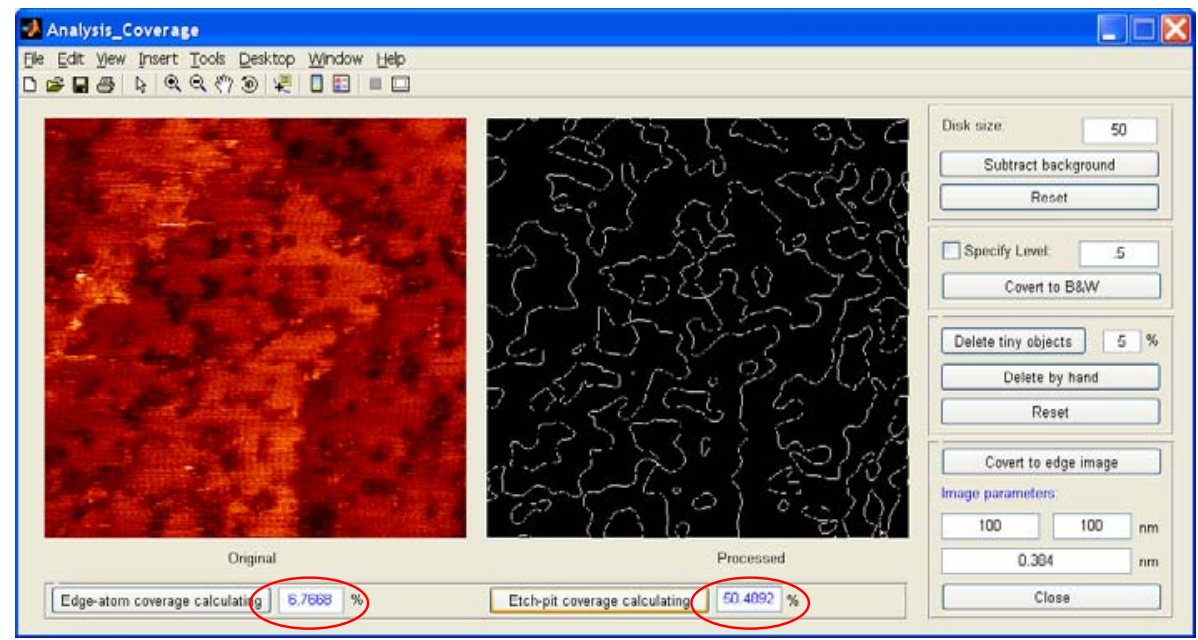

